\title{
Histopathologic Evidence of Tumor Regression in the Axillary Lymph Nodes of Patients Treated With Preoperative Chemotherapy Correlates With Breast Cancer Outcome
}

\author{
Lisa A. Newman, MD, MPH, FACS, Nat L. Pernick, MD, PhD, Volkan Adsay, MD, \\ Kathryn A. Carolin, MD, FACS, Philip I. Philip, MD, Susan Sipierski, PA-C, \\ David L. Bouwman, MD, FACS, Mary A. Kosir, MD, FACS, Michael White, MD, and \\ Daniel W. Visscher, MD
}

\begin{abstract}
Background: The benefits of primary tumor downstaging and assessment of chemoresponsiveness have resulted in expanded applications for induction chemotherapy. However, the pathologic evaluation and prognostic significance of response in preoperatively treated lymph nodes have not been defined.

Methods: The axillary lymph nodes of 71 patients with locally advanced breast cancer treated with induction chemotherapy were evaluated for histological evidence of tumor regression as defined by the presence of nodal fibrosis, mucin pools, or aggregates of foamy histiocytes.

Results: Complete pathologic response in the breast and axilla occurred in 10 patients (14\%); 19 $(26.8 \%)$ had evidence of tumor regression in 1 or more lymph nodes. Patients without nodal metastases and no evidence of tumor regression had the best outcome (median disease-free survival, 31.5 months; relapse rate, $27 \%$ ). Patients with residual nodal metastases and no evidence of treatment effect had the worst outcome (median disease-free survival, 19.8 months; relapse rate, $55 \%$ ). The median disease-free survival was 22.1 months, and the relapse rate was $32 \%$ for patients with histopathologic evidence of tumor regression in the axillary lymph nodes.

Conclusions: Detection of treatment effect in axillary lymph nodes after induction chemotherapy identifies a subset of patients with an outcome intermediate between that of completely nodenegative and node-positive patients. The axillary lymph nodes of patients receiving preoperative chemotherapy should be routinely analyzed for the presence of these features.
\end{abstract}

Key Words: Neoadjuvant chemotherapy_Axillary lymph nodes-Breast cancer.

Induction chemotherapy (CTX) is standard management for patients with locally advanced breast cancer. The benefits of tumor downstaging and the ability to assess tumor response to CTX in vivo have resulted in expanded applications of this treatment approach to patients with earlystage, resectable disease. ${ }^{1-6}$ CTX can sterilize axillary

Received March 25, 2002; accepted May 23, 2003.

From the Departments of Surgery (LAN, KAC, SS, DLB, MAK, MW), Medical Oncology (PIP), and Pathology (NLP, VA, DWV), Karmanos Cancer Institute and Wayne State University, Detroit, Michigan.

Address correspondence and reprint requests to: Lisa A. Newman, MD, MPH, FACS, University of Michigan Comprehensive Cancer Center, 3308 Cancer Center, 1500 East Medical Center Drive, Ann Arbor, MI 48109-0932; Fax: 734-647-9647; E-mail: lanewman@ med.umich.edu.

Published by Lippincott Williams \& Wilkins @ 2003 The Society of Surgical Oncology, Inc. nodal metastases in $25 \%$ to $30 \%$ of cases, ${ }^{4,7}$ and the extent of nodal disease retains prognostic value. ${ }^{8}$

However, pathologic assessment of the CTX effect on nodal metastases is poorly understood. Improved methods of evaluating lymph nodes in patients receiving preoperative CTX will facilitate the integration of lymphatic mapping into induction CTX protocols. The goal of this study was to correlate a predetermined profile of nodal CTX response features with outcome in a series of patients with locally advanced breast cancer.

\section{METHODS}

Histopathologic findings from surgical specimens of 71 patients with locally advanced breast cancer (stages IIB, IIIA, IIB and IV; limited or supraclavicular metas- 
tases) treated with preoperative CTX were evaluated. The study patients were treated between 1993 and 1999, and CTX regimens included doxorubicin in all patients. A standard level I/II axillary lymph node dissection was performed in all cases, and all patients received postoperative chest radiotherapy. Axillary lymph nodes were studied for evidence of metastases by serial sectioning and hematoxylin and eosin staining.

Evidence of tumor regression, or response to induction CTX, within an axillary lymph node was defined as the presence of nodal fibrosis, mucin pools, or large aggregates of foamy histiocytes. An example of nodal fibrosis and mucinous changes is shown in Fig. 1.

Statistical analysis was conducted with the $t$-test for continuous variables and the $\chi^{2}$ test for discrete variables. Survival was evaluated with the Kaplan-Meier method. Median follow-up was 29 months.

\section{RESULTS}

Clinical characteristics of the study population are listed in Table 1. The median patient age was 51 years. Most patients had invasive ductal carcinomas. Slightly more than half of all patients had T4 tumors, and more three quarters had either N1 or N2 disease at presentation.

Pathologic response findings are listed in Table 2. A complete pathologic response (no residual invasive disease in the breast specimen or in axillary lymph nodes) occurred in 10 patients (14\%); one of these patients had residual ductal carcinoma-in-situ in the breast tissue. Assessment of response to CTX in axillary lymph nodes revealed 12 patients with no axillary nodal disease and no evidence of prior nodal metastases that had responded

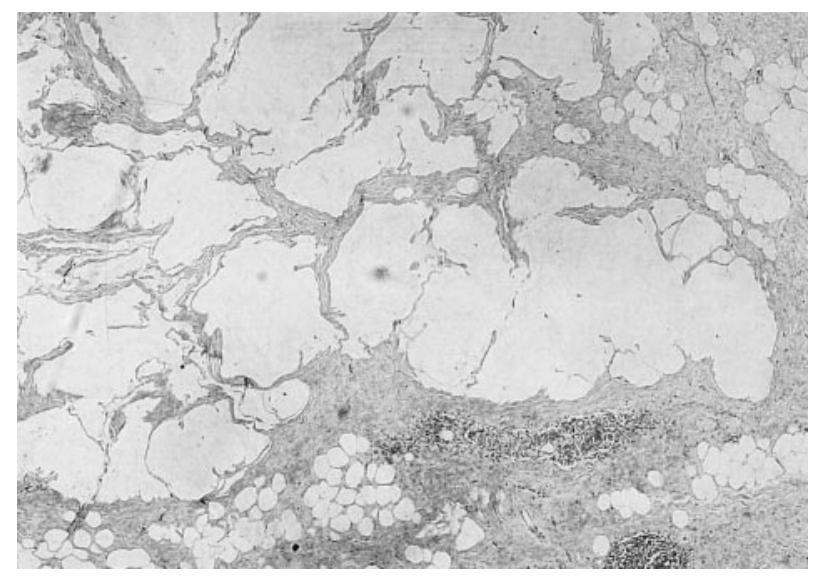

FIG. 1. Histopathology patterns of fibrosis and mucinous changes consistent with a treatment effect in the lymph node of a breast cancer patient.
TABLE 1. Clinical characteristics of the study patient population $(N=71$ patients $)$

\begin{tabular}{lc}
\hline \multicolumn{1}{c}{ Feature } & Data \\
\hline Median age, y & 51 \\
Race & \\
Black & $37(52 \%)$ \\
White & $34(48 \%)$ \\
AJCC disease stage & $6(8.5 \%)$ \\
IIB & $25(35.2 \%)$ \\
IIIA & $33(46.5 \%)$ \\
IIIB & $7(9.9 \%)$ \\
IV (limited/supraclavicular metastases) & \\
T category & $33(46.5 \%)$ \\
T3 & $38(53.5 \%)$ \\
T4 & \\
N category & $12(16.9 \%)$ \\
N0 & $47(66.2 \%)$ \\
N1 & $12(16.9 \%)$ \\
N2 & \\
Histology & $69(97 \%)$ \\
Invasive ductal & $2(3 \%)$ \\
Invasive lobular & \\
\hline
\end{tabular}

AJCC, American Joint Committee on Cancer, Fifth Edition.

to treatment. A total of 19 patients $(27 \%)$ had histological evidence of tumor regression in axillary lymph nodes; 9 of these patients also had residual viable lymph node metastases, and 10 had no evidence of viable axillary metastases.

As shown in Table 3, only tumor category at presentation was a statistically significant predictor of treatment failure. Twenty-seven percent of patients with T3 tumors at presentation experienced disease relapse, compared with $57.9 \%$ of patients with T4 tumors.

For the survival analysis, the patients with evidence of disease response in axillary lymph nodes were considered together in one group, regardless of whether or not

TABLE 2. Pathologic findings in breast and axillary specimens: response to chemotherapy

\begin{tabular}{lr}
\hline \multicolumn{1}{c}{ Pathologic findings } & $\begin{array}{c}\text { No. } \\
\text { Patients } \\
(\%)\end{array}$ \\
\hline $\begin{array}{l}\text { Complete response (no residual invasive disease; axillary } \\
\text { lymph nodes negative) }\end{array}$ & $10(14.1)$ \\
$\begin{array}{l}\text { Partial response ( } \geq 50 \% \text { decrease in primary tumor size) } \\
\text { No response/stable disease }(<50 \% \text { decrease in primary } \\
\text { tumor size) }\end{array}$ & $53(74.6)$ \\
$\begin{array}{l}\text { Axillary nodal pathology findings } \\
\text { No axillary metastases; no evidence of nodal tumor }\end{array}$ & $12(11.3)$ \\
$\quad$ regression & $10(14.1)$ \\
$\quad \begin{array}{l}\text { No axillary metastases; positive evidence of nodal } \\
\text { tumor regression }\end{array}$ & $9(12.7)$ \\
$\quad \begin{array}{l}\text { Positive axillary metastases; positive evidence of nodal } \\
\text { tumor regression }\end{array}$ & $39(54.9)$ \\
$\quad \begin{array}{l}\text { Positive axillary metastases; no evidence of nodal } \\
\text { tumor regression }\end{array}$ & \\
\hline
\end{tabular}

${ }^{a}$ Data are missing on one patient 
TABLE 3. Clinicopathologic features associated with disease relapse

\begin{tabular}{lcc}
\hline \multicolumn{1}{c}{ Feature } & $\begin{array}{c}\text { No. Relapses } \\
(\%)\end{array}$ & $P$ value \\
\hline Race & & \\
Black & $16 / 37(43.2)$ & .94 \\
White & $15 / 34(44.1)$ & \\
Tumor category at presentation & $9 / 33(27.3)$ & .009 \\
T3 & $22 / 38(57.9)$ & \\
T4 & & \\
Nodal status at presentation & $6 / 12(50)$ & .74 \\
N0 & $19 / 47(40.4)$ & \\
N1 & $6 / 12(50)$ & \\
N2 & $1 / 6(16.7)$ & \\
Stage at presentation & $8 / 25(32)$ & .10 \\
IIB & $17 / 33(51.5)$ & \\
IIIA & $5 / 7(71.4)$ & \\
IIIB & & \\
IV & $25 / 49(50)$ & .08 \\
Pathologic nodal status & $6 / 21(28.6)$ & \\
Positive & & \\
Negative & $4 / 10(40)$ & .91 \\
Pathologic response & $23 / 53(43.4)$ & \\
Complete response & $4 / 8(50)$ & \\
Partial response & & \\
No response & & \\
Histopathologic evidence of tumor & & \\
regression in axillary lymph & & \\
nodes & & \\
Present & & \\
Absent & & \\
\hline
\end{tabular}

viable metastases were also present. These subsets were considered together because of the small sample size (nine patients) of cases with the mixed pattern of tumor regression in the presence of residual metastases. Relapse rates and median disease-free survival times for the remaining patient subsets based on axillary pathology findings are listed in Table 4. The small sample sizes preclude meaningful statistical testing, but a trend was seen for improved outcome in the subsets of patients who either were completely node negative or seemed to have been downstaged to node negativity because of a treatment effect on axillary metastases, compared with the subset of patients who had residual viable axillary me-

TABLE 4. Outcome correlated with pathologic findings in axillary lymph nodes

\begin{tabular}{ccc}
\hline Axillary histopathology & $\begin{array}{c}\text { Relapse } \\
\text { rate }\end{array}$ & $\begin{array}{c}\text { Median } \\
\text { DFS (mo) }\end{array}$ \\
\hline $\begin{array}{c}\text { No nodal metastases; no evidence of nodal } \\
\text { disease regression } \pm \text { axillary metastases } \\
(\mathrm{n}=12)\end{array}$ & $27 \%$ & 31.5 \\
$\begin{array}{c}\text { Evidence of regression in axillary nodes; } \\
\text { metastases present }(\mathrm{n}=19)\end{array}$ & $32 \%$ & 22.1 \\
$\begin{array}{c}\text { Axillary metastases present; no evidence } \\
\text { of nodal disease regression }(\mathrm{n}=39)\end{array}$ & $55 \%$ & 19.8 \\
\hline
\end{tabular}

DFS, disease-free survival. tastases. However, the subset of downstaged node-negative patients had a higher relapse rate and shorter disease-free survival compared with the completely nodenegative patients.

\section{DISCUSSION}

\section{History of Induction CTX: Locally Advanced Breast Cancer}

Primary CTX followed by definitive breast and axillary is now well established as standard management for patients with locally advanced breast cancer. ${ }^{1,9}$ Initial reservations regarding this treatment sequence were based on concerns that delayed surgery might result in increased surgical morbidity and loss of a "window of opportunity" to obtain durable locoregional disease control with surgical intervention. These concerns regarding the surgical management of locally advanced disease have been largely answered by studies demonstrating that approximately $80 \%$ of patients will experience significant shrinkage of their breast tumors in response to primary CTX, thereby facilitating subsequent surgery. In contrast, only $2 \%$ to $3 \%$ of patients will exhibit progressive disease. Furthermore, studies of preoperative versus postoperative CTX for locally advanced breast cancer patients have demonstrated that surgical complication rates are unaffected by the neoadjuvant CTX sequence. ${ }^{10}$

An additional area of concern has been the issue of preoperative CTX effect on axillary lymph nodes. The presence versus absence of axillary nodal metastases remains the most powerful prognostic feature available in stratifying breast cancer patients for risk of relapse. Physical examination is notoriously inaccurate in predicting the status of axillary lymph nodes, and even the most sophisticated soft tissue imaging modalities, such as ultrasound, computerized tomography, and magnetic resonance imaging, are unable to replace surgical resection and histopathologic examination. ${ }^{11}$ It was therefore necessary for early studies of neoadjuvant CTX to address the question of whether the CTX effect on axillary lymph node metastases might eliminate valuable prognostic information. McCready et al., ${ }^{8}$ however, reported on survival rates for 136 patients with locally advanced breast cancer from the University of Texas M. D. Anderson Cancer Center, all of whom received induction doxorubicin, fluorouracil, and cyclophosphamide before undergoing modified radical mastectomy. These investigators found that the extent of residual axillary nodal metastases correlated well with outcome and was an independent predictor of survival even after controlling for tumor stage at presentation, tumor grade, and menopausal status. 
The promising results of surgical downstaging with neoadjuvant CTX led to successful investigations of breast-conservation therapy for patients with locally advanced breast cancer. Singletary et al. ${ }^{12}$ conducted a feasibility study of breast-conserving therapy in a series of locally advanced breast cancer patients treated with preoperative CTX followed by mastectomy, and scrutiny of the pathology specimens revealed that $23 \%$ of patients had sufficient primary tumor shrinkage that a lumpectomy could have been successfully performed. Other studies have subsequently confirmed the oncological safety of breast preservation in patients with locally advanced breast cancer who are adequately downstaged by preoperative CTX, and local recurrence rates are comparable to those seen for lumpectomy in early-stage breast cancer patients. ${ }^{13,14}$

The conceptual advantage of in vivo assessment of primary tumor response to the selected CTX regimen is another benefit derived from the neoadjuvant CTX approach. When systemic therapy is administered in the postoperative setting, the patient and oncologist cannot determine whether micrometastases are resistant to the selected regimen until distant metastases become clinically overt in long-term follow-up. Unfortunately, however, stage IV breast cancer is notoriously refractory to successful treatment. Preoperative CTX allows the clinician to evaluate the effectiveness of therapy within several weeks as the initial one or two cycles of CTX are administered. If the desired response is not seen, then switching to a non-cross-resistant regimen may be considered. This approach may minimize exposing the patient to the cumulative toxicities of an ineffective regimen.

Kuerer et al. ${ }^{15}$ confirmed the validity of tumor response to induction CTX as a surrogate marker of treatment effect on micrometastases in a survival study of breast cancer patients with complete pathologic response to doxorubicin-based preoperative CTX. Out of nearly 400 locally advanced breast cancer patients treated on prospective treatment trials, $12 \%$ had no evidence of residual invasive disease in their surgical specimens. With a median follow-up of nearly 5 years, these complete responders had a 5-year disease-free survival of $87 \%$, compared with only $58 \%$ for those with less than a complete response, and this difference in outcome was statistically significant. As the armamentarium of CTX combinations with effectiveness against breast tumors expands, additional crossover regimens for patients with resistant disease will be more readily available, and the pool of complete responders should enlarge in parallel. The advent of the taxanes, gemcitabine, and trastuzumab
(Herceptin; Genentech, South San Francisco, CA) is quite promising in this regard.

\section{Induction CTX and Early-Stage/Resectable Breast Cancer}

The excellent results of induction CTX for patients with locally advanced breast cancer have led to application of this approach to the setting of early-stage disease. The National Surgical Adjuvant Breast Project (NSABP) B-18 study ${ }^{2,3}$ has conclusively proven the safety of preoperative CTX in a prospective trial that randomized patients with operable stage I to III disease to receive preoperative versus postoperative CTX; it found overall survival equivalence between the two arms of the study. This study had striking similarities to the Kuerer et al. report ${ }^{15}$; a complete pathologic response occurred in $13 \%$ of the patients receiving doxorubicin-based preoperative CTX, and disease-free survival was $85.7 \%$ for this subset, which was significantly better than the survival rates of patients with a lesser response. Ongoing trials through the NSABP and other institutions are evaluating combinations of doxorubicin, paclitaxel, docetaxel, and vinorelbine in the preoperative setting for patients with early-stage resectable breast cancer.

\section{Induction CTX and Effect on Axillary Metastases}

Clearly, there will be an increasing population of patients with early-stage breast cancer who will be receiving systemic therapy before undergoing definitive surgery. A more thorough pathologic assessment of treatment effect on axillary lymph nodes is therefore warranted as we attempt to integrate minimally invasive approaches to axillary surgery into induction CTX algorithms and as we attempt to identify patients who may require additional CTX in the postoperative setting.

Currently, routine pathology practice is to perform basic hematoxylin and eosin staining of axillary nodal sections to identify viable metastases in patients with breast cancer, regardless of whether or not preoperative CTX has been delivered. The ability of neoadjuvant CTX to successfully downstage the axilla has been conclusively established, yet pathologic assessment of treatment response after neoadjuvant CTX is not routinely reported. Distinguishing patients who never had nodal metastases from those who had disease successfully eliminated by preoperative CTX is challenging, but it may have prognostic value and therapeutic implications.

In the NSABP B-18 study, ${ }^{3}$ axillary nodal metastases were found in $57 \%$ of patients randomized to receive postoperative CTX, compared with only $41 \%$ of patients receiving preoperative CTX $(P<.001)$. Furthermore, data from the M. D. Anderson Cancer Center ${ }^{4,7}$ have 
shown that among patients with fine-needle aspirationproven axillary nodal metastases at presentation, $23 \%$ to $30 \%$ will be completely node negative when axillary nodal dissection is performed after induction CTX.

Preliminary data from studies of postoperative, adjuvant CTX have indicated that patients with axillary nodal metastases may benefit from receiving sequential doxorubicin-based CTX and a taxane. ${ }^{16}$ Other investigators ${ }^{17}$ have reported that axillary nodal downstaging may be an even more powerful prognostic feature than primary breast tumor response in patients treated with preoperative CTX. It may therefore be important in planning therapeutic strategy to identify the patients who experienced CTX-induced eradication of nodal metastases. Outcome for these patients may be optimized by postoperative delivery of additional CTX cycles and, possibly, multiple-regimen therapies.

Another issue driving the importance of assessing pathologic response to preoperative CTX in the axilla is the increasingly popular technology of lymphatic mapping and sentinel lymph node biopsy. This minimally invasive means of evaluating the axilla is an extremely promising modality for accurately staging breast cancer patients while avoiding the morbidity of a full axillary lymph node dissection. However, the accuracy of sentinel lymph node biopsy in the setting of patients treated with induction CTX is not yet fully understood, because we do not know whether preoperative CTX will exert a uniform effect on all axillary nodal metastases. Falsenegative rates for sentinel lymph node biopsy in patients receiving neoadjuvant CTX have been reported to range from $0 \%$ to $33 \%$ in various studies, ${ }^{18-24}$ and identification of the sentinel lymph node may be more difficult because of the fibrotic reaction that may occur in axillary soft tissues. Development of a standardized approach for pathologically evaluating the CTX effect on axillary nodes will be essential as lymphatic mapping becomes incorporated into future preoperative CTX protocols.

Despite the numerous reported studies of neoadjuvant CTX and the clear evidence that the extent of pathologic response is a valid surrogate marker for chemosensitivity in micrometastases, the pathologic assessment of treatment response in breast and axillary tissue has not been standardized. The presence versus the absence of treatment effect within axillary lymph nodes may be particularly useful information. The ability to stratify preoperatively treated patients by node negativity versus positivity, as well as by whether the nodes were downstaged, may influence decisions regarding delivery of additional postoperative CTX.

Our study demonstrated that specific patterns of pathologic findings within the axillary lymph nodes of patients receiving induction CTX are reliable indicators of treatment response. These features include fibrosis within lymph nodes, the presence of mucin pools, and large aggregates of foamy histiocytes. As expected, stratification of breast cancer patients receiving neoadjuvant CTX into different categories on the basis of the presence versus absence of these features identified subsets of patients with varying degrees of risk for disease relapse. The patients with the best outcome were those patients with no axillary metastases and no evidence of treatment response. These patients were probably node negative at time of diagnosis and probably had the lowest burden of micrometastatic disease. At the other end of the spectrum, patients with residual viable axillary metastases had the worst outcome. These patients probably had the most advanced disease at diagnosis and micrometastases that were at resistant to systemic treatment. In the middle of the spectrum are the patients with or without residual axillary metastases at the time of surgery but who also show evidence of treatment response; these patients had an outcome intermediate between the completely node-negative patients and the node-positive patients. This subset of patients probably had axillary disease at time of diagnosis, but the disease was at least partially responsive to the selected CTX regimen. These patients probably have a greater micrometastatic burden compared with the completely node-negative patients without treatment effect. Conversely, the chemosensitivity of their disease defines them as a better prognostic subset compared with patients with residual nodal metastases and no treatment effect.

In summary, we believe that pathologic evaluation of axillary lymph nodes in patients receiving preoperative CTX should routinely include assessment of tumor response patterns. This information may guide the clinician regarding determination of CTX effectiveness and, more importantly, may better identify patients with resistant disease. This histopathologic response information may also facilitate the interpretation of sentinel lymph node biopsy in patients treated with induction CTX. Future studies of induction CTX and histopathologic assessment of the treatment effect in regional nodes, as well as correlation with the number of CTX cycles required to produce this effect, are warranted.

\section{REFERENCES}

1. Kuerer HM, Newman LA, Smith TL, et al. Clinical course of breast cancer patients with complete pathologic primary tumor and axillary lymph node response to doxorubicin-based neoadjuvant chemotherapy. J Clin Oncol 1999;17:460-9.

2. Fisher B, Brown A, Mamounas E, et al. Effect of preoperative chemotherapy on local-regional disease in women with operable 
breast cancer: findings from the National Surgical Adjuvant Breast and Bowel Project B-18. J Clin Oncol 1997;15:2483-93.

3. Fisher B, Wolmark N, Mamounas E, et al. Effect of preoperative chemotherapy on the outcome of women with operable breast cancer. J Clin Oncol 1998;16:2672-85.

4. Newman LA, Buzdar AU, Singletary SE, et al. A prospective trial of preoperative chemotherapy in resectable breast cancer: predictors of breast-conservation therapy feasibility. Ann Surg Oncol 2002;9:228-34.

5. Wolff AC, Davidson NE. Primary systemic therapy in operable breast cancer. J Clin Oncol 2000;18:1558-69.

6. Makris A, Powles TJ, Ashley SE, et al. A reduction in the requirements for mastectomy in a randomized trial of neoadjuvant chemoendocrine therapy in primary breast cancer. Ann Oncol 1998; 9:1179-84.

7. Kuerer HM, Sahin AA, Hunt KK, et al. Incidence and impact of documented eradication of breast cancer axillary lymph node metastases before surgery in patients treated with neoadjuvant chemotherapy. Ann Surg 1999;230:72-8.

8. McCready DR, Hortobagyi GN, Kau SW, Smith TL, Buzdar AU, Balch CM. The prognostic significance of lymph node metastases after preoperative chemotherapy for locally advanced breast cancer. Arch Surg 1989;124:21-5.

9. Hunt KK, Ames FC, Singletary SE, Buzdar AU, Hortobagyi GN. Locally advanced noninflammatory breast cancer. Surg Clin North Am 1996;76:393-410.

10. Broadwater JR, Edwards MJ, Kuglen C, Hortobagyi GN, Ames FC, Balch CM. Mastectomy following preoperative chemotherapy. Ann Surg 1991;213:126-9.

11. Hansen N, Morrow M. Current status of axillary node dissection. In: Singletary SE, ed. M.D. Anderson Solid Tumor Oncology Series: Breast Cancer. New York: Springer, 1998: 155-70.

12. Singletary SE, McNeese MD, Hortobagyi GN. Feasibility of breast conservation surgery after induction chemotherapy for locally advanced breast cancer. Cancer 1992;69:2849-52.

13. Peoples G, Heaton K, Booser D, et al. Breast conservation therapy for large primary and locally advanced breast cancers after induction chemotherapy (abstract 25). Abstract presented at: Society of Surgical Oncology 51st Annual Cancer Symposium; March 15, 1998; San Diego, CA.

14. Bonnadonna G, Veronesi U, Brambilla C, et al. Primary che- motherapy to avoid mastectomy in tumors with diameters of three centimeters or more. J Natl Cancer Inst 1990;82:153945 .

15. Kuerer HM, Newman LA, Smith TL, et al. Clinical course of breast cancer patients with complete pathologic primary tumor and axillary lymph node response to doxorubicin-based neoadjuvant chemotherapy. J Clin Oncol 1999;17:460-9.

16. Abrams JS. Adjuvant therapy for breast cancer-results from the USA consensus conference. Breast Cancer 2001;8:298-304.

17. Rouzier R, Extra JM, Klijanienko J, et al. Incidence and prognostic significance of complete axillary downstaging after chemotherapy in breast cancer patients with $\mathrm{T} 1$ to $\mathrm{T} 3$ tumors and cytologically proven axillary metastatic lymph nodes. J Clin Oncol 2002;20: 1304-10.

18. Breslin TM, Cohen L, Sahin A, et al. Sentinel lymph node biopsy following neoadjuvant chemotherapy for breast cancer. J Clin Oncol 2000;18:348-86.

19. Haid A, Tausch C, Lang A, et al. Is sentinel lymph node biopsy reliable and indicated after preoperative chemotherapy in patients with breast carcinoma? Cancer 2001;92:1080-4.

20. Nason KS, Anderson BO, Byrd DR, et al. Increased false negative sentinel node biopsy rates after preoperative chemotherapy for invasive breast carcinoma. Cancer 2000;89:2187-94.

21. Mamounas E. Sentinel node biopsy following neoadjuvant chemotherapy in breast cancer: results from NSABP B-27 (abstract). Abstract presented at: Proceedings of the American Society of Clinical Oncology; May 19, 2002; Orlando, FL.

22. Balch GC, Mithani SK, Richards K, Beauchamp RD, Kelley MC. Prospective evaluation of the accuracy of sentinel lymphadenectomy after preoperative chemotherapy for locally advanced breast cancer (abstract 66). Abstract presented at: Society of Surgical Oncology 55th Annual Cancer Symposium; March 16, 2002; Denver, CO.

23. Miller AR, Thomason VE, Yeh IT, et al. Analysis of sentinel lymph node mapping with immediate pathologic review in patients receiving preoperative chemotherapy for breast carcinoma. Ann Surg Oncol 2002;9:243-7.

24. Stearns V, Ewing CA, Slack R, et al. Sentinel lymphadenectomy after neoadjuvant chemotherapy for breast cancer may reliably represent the axilla except for inflammatory breast cancer. Ann Surg Oncol 2002;9:235-42. 